\title{
The contribution of galaxies to the UV ionising background and the evolution of the Lyman forest
}

\author{
S. Bianchi ${ }^{1, \star}$, S. Cristiani ${ }^{2,3}$, and T.-S. Kim ${ }^{1}$ \\ 1 European Southern Observatory, Karl-Schwarzschild-Strasse 2, 85748 Garching, Germany \\ 2 Space Telescope European Coordinating Facility, ESO, Karl-Schwarzschild-Strasse 2, 85748 Garching, Germany \\ 3 Dipartimento di Astronomia dell'Università di Padova, Vicolo dell'Osservatorio, 35122 Padova, Italy
}

Received 11 May 2001 / Accepted 27 June 2001

\begin{abstract}
We have modelled the evolution of the number of Ly $\alpha$ absorbers with redshift, resulting from the evolution of the ionising background and the Hubble expansion. The contribution of quasars (QSOs) and galaxies to the H I-ionising UV background has been estimated. The QSOs emissivity is derived from recent fits of their luminosity function. The galaxy emissivity is computed using a stellar population synthesis model, with a starformation history scaled on observations of faint galaxies at $\lambda \geq 1500 \AA$. We allow for three values of the fraction of ionising photons that can escape the interstellar medium, $f_{\mathrm{esc}}=0.05,0.1$ and 0.4 . The Intergalactic Medium is modelled as made of purely-absorbing clouds with the distribution in redshift and column density obtained from QSOs absorption lines. For the adopted values of $f_{\text {esc }}$, the contribution of galaxies to the ionising UV background is comparable or greater than that of QSOs. Accounting for the contribution of clouds to the UV emission, all models with $f_{\text {esc }} \lesssim 0.1$ provide an ionising flux compatible with local and high- $z$ determination, including those with a pure QSOs background. The observed $z \sim 1$ break in the evolution can be better explained by a dominant contribution from galaxies. We find that models in $\Lambda$-cosmology with $\Omega_{\mathrm{m}}=0.3, \Omega_{\Lambda}=0.7$ describe the flat absorbers evolution for $z \lesssim 1.0$ better than models for $\Omega_{\mathrm{m}}=1.0$.
\end{abstract}

Key words. radiative transfer - diffuse radiation - intergalactic medium - cosmology: theory - quasar: absorption lines - ultraviolet: galaxies

\section{Introduction}

The evolution of the Lyman forest is governed by two main factors: the Hubble expansion and the the metagalactic UV background. At high redshift the expansion, which tends to increase the ionisation of the matter, and the UV background, increasing or non-decreasing with decreasing redshift, work in the same direction and cause a steep evolution of the number of lines with $z$. At low redshift, the UV background starts to decrease with decreasing redshift, due to the reduced number of ionising sources, and this effect counteracts the Hubble expansion. As a result the evolution of the number of lines slows down.

In a recent study of the evolution of the Lyman forest Kim et al. (2001) have shown that the number of Ly $\alpha$ lines per unit redshift, $\mathrm{d} N / \mathrm{d} z$, is well described by a double power-law with a break at $z \sim 1$. For column densities in the interval $N_{\mathrm{H}_{\mathrm{I}}}=10^{13.64-16} \mathrm{~cm}^{-2}, \mathrm{~d} N / \mathrm{d} z \propto(1+$ $z)^{2.19 \pm 0.27}$ at $1.5<z<4$, while $\mathrm{d} N / \mathrm{d} z \propto(1+z)^{0.16 \pm 0.16}$ at $z<1$ (Weymann et al. 1998).

Send offprint requests to: S. Bianchi,

e-mail: sbianchi@eso.org

* Fellow of the European Community Research and Training Network: The Physics of the Intergalactic Medium.
Recent numerical simulations have been remarkably successful in reproducing the observed evolution (see, for example Davé et al. 1999; Machacek et al. 2000), leaving little doubt about the general interpretation of the phenomenon. However the same simulations predict the break in the $\mathrm{d} N / \mathrm{d} z$ power-law at a redshift $z \sim 1.8$ which appears too high in the light of the new results of Kim et al. (2001). This suggests that the UV background implemented in the simulations is not completely correct: QSOs have been considered as the main source of ionising photons, and, since their space density drops below $z \sim 2$, so does the UV background. However, galaxies can produce a non-negligible ionising flux too, perhaps more significant than previously assumed, as shown by recent measurements by Steidel et al. (2001). The galaxy contribution can keep the UV background relatively high until at $z \sim 1$ the global star formation rate in the Universe quickly decreases, determining the change in the number density of lines.

In this paper we recompute the contribution of QSOs and galaxies to the UV background following the recipes of Madau (1991, 1992), Haardt \& Madau (1996) and Madau et al. (1998) (Sect. 2). In Sect. 3 the results are compared with constraints on the UV background derived from the 
proximity effect and the $\mathrm{H} \alpha$ emission of high galactic latitude clouds. In Sect. 4 the evolution of the number of Ly $\alpha$ lines per unit redshift is computed according to a simple analytical model for different relative contributions of galaxies and QSOs. In Sect. 5 we summarise the results and discuss some consequences of them in terms of present and future observations.

All the computations are carried out for two flat cosmologies: an Einstein-De Sitter cosmology $\left(\Omega_{\mathrm{m}}=1\right.$, $\left.\Omega_{\Lambda}=0\right)$ and a $\Lambda$ cosmology $\left(\Omega_{\mathrm{m}}=0.3, \Omega_{\Lambda}=0.7\right)$. We have adopted $H_{0}=70 \mathrm{~km} \mathrm{~s}^{-1} \mathrm{Mpc}^{-1}$ (Freedman et al. 2001) throughout and scaled to this value the data derived from the literature.

\section{The ultraviolet background}

The mean specific intensity of the Ultraviolet background $J\left(\nu_{\mathrm{obs}}, z_{\mathrm{obs}}\right)$, as seen at a frequency $\nu_{\mathrm{obs}}$ by an observer at redshift $z_{\mathrm{obs}}$, can be derived from

$$
\begin{aligned}
J\left(\nu_{\mathrm{obs}}, z_{\mathrm{obs}}\right)= & \frac{1}{4 \pi} \int_{z_{\mathrm{obs}}}^{\infty} \frac{\left(1+z_{\mathrm{obs}}\right)^{3}}{(1+z)^{3}} \epsilon(\nu, z) \\
& \times \quad \mathrm{e}^{-\tau_{\text {eff }}\left(\nu_{\mathrm{obs}}, z_{\mathrm{obs}}, z\right)} \frac{\mathrm{d} l}{\mathrm{~d} z} \mathrm{~d} z,
\end{aligned}
$$

where $\nu=\nu_{\mathrm{obs}}(1+z) /\left(1+z_{\mathrm{obs}}\right), \epsilon(\nu, z)$ is the proper space-averaged volume emissivity, $\tau_{\text {eff }}\left(\nu_{\mathrm{obs}}, z_{\mathrm{obs}}, z\right)$ is the effective optical depth at $\nu_{\mathrm{obs}}$ of the Intergalactic Medium (IGM) between redshifts $z_{\text {obs }}$ and $z$, and $\mathrm{d} l / \mathrm{d} z$ the proper line element (Madau 1991, 1992; Haardt \& Madau 1996).

The emissivity $\epsilon(\nu, z)$ should include a contribution both from direct sources of UV radiation (e.g. QSOs and galaxies) and from the IGM clouds themselves, through continuum radiative recombination of the gas (Haardt \& Madau 1996). For the sake of simplicity, we consider here the case of a purely absorbing IGM, thus omitting radiative recombination. The effect of this omission will be discussed later.

The line element can be written as

$$
\frac{\mathrm{d} l}{\mathrm{~d} z}=\frac{c}{H(z)(1+z)},
$$

where $c$ is the velocity of light and $H(z)=H_{0}\left[\Omega_{\mathrm{m}}(1+\right.$ $\left.z)^{3}+\Omega_{\Lambda}\right]^{1 / 2}$ is the Hubble parameter for a flat universe $\left(\Omega=\Omega_{\mathrm{m}}+\Omega_{\Lambda}=1\right)$.

\subsection{Opacity}

The effective optical depth $\tau_{\text {eff }}$ through the IGM is defined as $\mathrm{e}^{-\tau_{\text {eff }}}=\left\langle\mathrm{e}^{-\tau}\right\rangle$, where the mean is taken over all the lines of sight from the redshift of interest. For a Poisson distribution of discrete absorbers (Paresce et al. 1980; Moller \& Jakobsen 1990; Madau 1991, 1992),

$$
\begin{aligned}
& \tau_{\text {eff }}\left(\lambda_{\text {obs }}, z_{\text {obs }}, z\right)= \\
& \qquad \int_{z_{\text {obs }}}^{z} \mathrm{~d} z^{\prime} \int_{0}^{\infty} \mathrm{d} N_{\mathrm{H}} f\left(N_{\mathrm{HI}}, z^{\prime}\right)\left(1-\mathrm{e}^{-\tau\left(\lambda^{\prime}\right)}\right),(3)
\end{aligned}
$$

where $f\left(N_{\mathrm{H}}, z^{\prime}\right)=\partial^{2} N / \partial N_{\mathrm{H}_{\mathrm{I}}} \partial z^{\prime}$ is the distribution of absorbers as a function of redshift and column density of the atomic hydrogen $N_{\mathrm{H}}$, and $\tau\left(\lambda^{\prime}\right)$ is the optical depth of an individual cloud for ionising radiation at a wavelength $\lambda^{\prime}=\lambda_{\text {obs }}\left(1+z_{\text {obs }}\right) /(1+z)$. For $228 \AA<\lambda^{\prime} \leq 912 \AA$, the main contribution to absorption of UV photons is given by the ionisation of $\mathrm{HI}$, therefore

$\tau\left(\lambda^{\prime}\right)=\tau_{\mathrm{H} \text { I }}\left(\lambda^{\prime}\right)=N_{\mathrm{H}_{\mathrm{I}}} \sigma_{\mathrm{H} \text { I }}\left(\frac{\lambda^{\prime}}{912 \AA}\right)^{3}$,

where $\sigma_{\mathrm{H} \text { I }}=6.3 \times 10^{-18} \mathrm{~cm}^{-2}$ is the photo-ionisation cross-section at the Lyman limit for H I (Osterbrock 1989).

The ionisation of He I at $504 \AA$ is not considered: He I being almost completely ionised, its contribution to the total opacity is negligible (Haard \& Madau 1996). For completeness, we have included the contribution of He II ionisation to the opacity, although it does not affect the results presented in this paper. He II is ionised for $\lambda^{\prime} \leq 228 \AA$ : thus,

$\tau\left(\lambda^{\prime}\right)=\tau_{\mathrm{H} \mathrm{I}}+N_{\text {He II }} \sigma_{\text {He II }}\left(\frac{\lambda^{\prime}}{228 \AA}\right)^{3}$,

with $\sigma_{\text {He II }}=1.58 \times 10^{-18} \mathrm{~cm}^{-2}$ (Osterbrock 1989). The column density $N_{\text {He II }}$ can be derived from $N_{\mathrm{H} \mathrm{I}}$ by solving the radiative transfer within a cloud. When clouds are optically thin at $228 \AA$, a simple solution can be found (Haard \& Madau 1996):

$N_{\mathrm{He} \text { II }} \approx 1.8 N_{\mathrm{H} \text { I }} \frac{J(912 \AA)}{J(228 \AA)}$.

While Eq. (6) does not hold for optically thick clouds, its use in the optically thick case can still provide correct estimates for the cosmic opacity, when $\lambda_{\text {obs }}>228 \AA$ (Haard \& Madau 1996). Since the He II contribution to $\tau_{\text {eff }}$ depends on the UV background, we have solved Eq. (1) iteratively for every value of $z_{\mathrm{obs}}$.

For the redshift and column density distribution of absorption lines we have adopted the usual form

$f\left(N_{\mathrm{HI}}, z\right)=\left(\frac{A}{10^{17}}\right)\left(\frac{N_{\mathrm{H} \mathrm{I}}}{10^{17} \mathrm{~cm}^{-2}}\right)^{-\beta}(1+z)^{\gamma}$.

Fits of the absorption line distribution show that the in$\operatorname{dex} \beta$ varies for different ranges of $N_{\mathrm{H}}$ (Fardal et al. 1998; Kim et al. 2001). However, it is possible to describe the cloud distribution with $\beta=1.46$ over several decades in $N_{\mathrm{H} \text { I }}$ (Petitjean et al. 1993). For simplicity, we have adopted this single value over the whole column density range considered here. Kim et al. (2001) combined high resolution VLT/UVES observations of 3 QSOs with literature data and derived a line number density per unit redshift $\mathrm{d} N / \mathrm{d} z=9.06(1+z)^{2.19}$, for the column density range $N_{\mathrm{H} \text { I }}=10^{13.64-16} \mathrm{~cm}^{-2}$ and $z>1.5$. At lower redshifts, HST observations from the QSO absorption line key project show a slower evolution with redshift, with $\mathrm{d} N / \mathrm{d} z=34.7(1+z)^{0.16}$ (Weymann et al. 1998). The change in evolution, according to the results of Weymann et al. (1998) and Kim et al. (2001), occurs at $z \sim 1$. In this paper, we use $\gamma=2.19$ to describe the 
Table 1. Parameters adopted for the distribution of absorbing clouds (Eq. (7)).

\begin{tabular}{lllll}
\hline$A$ & $\beta$ & $\gamma$ & $N_{\mathrm{H}_{\mathrm{I}}} / \mathrm{cm}^{-2}$ & \\
\hline 0.50 & 1.46 & 0.16 & $10^{13}-1.58 \times 10^{17}$ & $z \leq 1$ \\
0.13 & 1.46 & 2.19 & $10^{13}-1.58 \times 10^{17}$ & $z>1$ \\
0.17 & 1.46 & 1.55 & $1.58 \times 10^{17}-10^{20}$ & \\
\hline
\end{tabular}

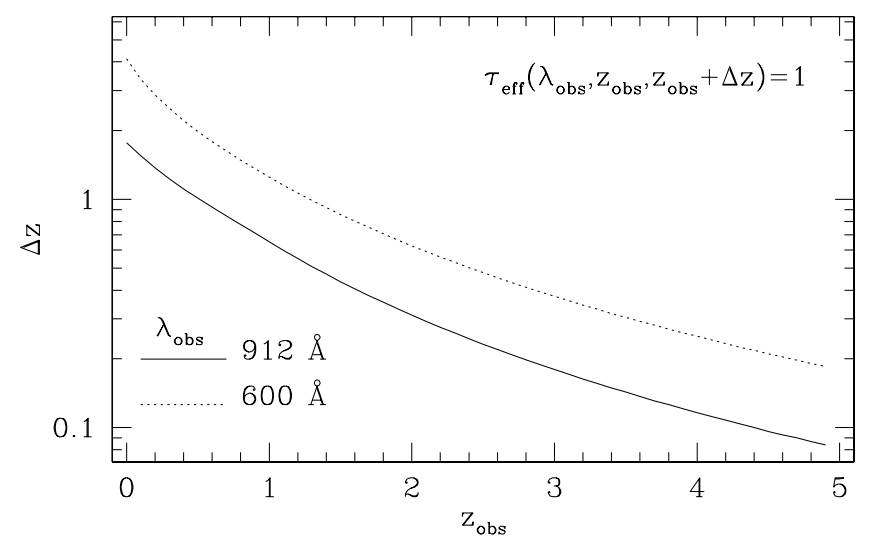

Fig. 1. Distance in redshift $\Delta z$ between an observer at $z_{\text {obs }}$ and a point at $z=z_{\mathrm{obs}}+\Delta z$ for which $\tau_{\mathrm{eff}}\left(\lambda_{\mathrm{obs}}, z_{\mathrm{obs}}, z\right)=1$, as a function of $z_{\text {obs }}$. Plots are shown for radiation observed at the Lyman edge and for $\lambda_{\text {obs }}=600 \AA$. The plot does not include the contribution to the opacity of the He II ionisation, that will affect the results at $600 \AA$. Assuming $J(912 \AA) / J(228 \AA)=$ 50 (the minimum value in our simulations, when only QSOs contribute to the background), $\Delta z$ at $600 \AA$ will decrease to $\approx 2$ in the range $0<z<0.5$.

redshift evolution of the Ly $\alpha$ forest $\left(10^{13} \leq N_{\mathrm{HI}} / \mathrm{cm}^{-2} \leq\right.$ $\left.1.58 \times 10^{17}\right)$ at $z>1$, and $\gamma=0.16$ at $z \leq 1$. By choosing $A=0.13$ and $A=0.50$, the integral of Eq. (7) over $N_{\mathrm{H}}=10^{13.64-16} \mathrm{~cm}^{-2}$ reproduces the results of Kim et al. (2001) and Weymann et al. (1998), respectively. The distribution of Lyman Limit systems is derived in an analogous way from Storrie-Lombardi et al. (1994). For $1.58 \times 10^{17} \leq N_{\mathrm{HI}} / \mathrm{cm}^{-2} \leq 10^{20}$, we use $A=0.17$ and $\gamma=1.55$. The parameters adopted for the distribution of absorbers are summarized in Table 1.

As already pointed out by many authors (Madau 1991, 1992; Haardt \& Madau 1996), the UV background becomes more dominated by local sources as the redshift increases. This can be seen in Fig. 1, where we show the distance in redshift $\Delta z=z-z_{\mathrm{obs}}$ corresponding to an effective optical depth $\tau_{\text {eff }}\left(\lambda_{\text {obs }}, z_{\text {obs }}, z\right)=1$, as a function of $z_{\mathrm{obs}}$. For radiation at $\lambda_{\mathrm{obs}}=912 \AA \Delta z$ decreases from 1.8 at $z_{\text {obs }}=0$ to 0.08 at $z_{\text {obs }}=5$. The same trend can be seen for $\lambda_{\text {obs }}=600 \AA$ but with larger values of $\Delta z$ (less absorption), because of the dependence of the $\mathrm{H}$ I ionisation cross-section on the wavelength. Since only radiation from local sources can easily reach $z_{\text {obs }}$, it is not necessary to compute the integral in Eq. (1) up to $z=\infty$ (or to the maximum $z$ for which UV emitting sources are available). We have used in our calculation $z_{\max }=5$.
The absorber distribution we have adopted produces opacities that are very similar to those of Haardt \& Madau (1996). Fardal et al. (1998, see also Shull et al. 1999) have derived values for $A, \beta$ and $\gamma$ by fitting the distribution of absorption lines in several ranges of column density. The opacity provided by their model is smaller than the one presented here. For example, at $z_{\mathrm{obs}}=3$ we reach $\tau_{\text {eff }}(912 \AA)=1$ for $\Delta z=0.18$, while it is $\Delta z=0.25$ for model A2 in Fardal et al. (1998). For the same emissivities, the opacity of Fardal et al. (1998) will result in a UV background higher than ours. The difference increases with $z_{\mathrm{obs}}$ and reaches 0.1 dex at $z_{\mathrm{obs}}=3$.

\subsection{QSO emissivity}

The QSO contribution to the UV emissivity has been derived from the QSO luminosity function, for which we have adopted the double power-law Pure Luminosity Evolution model (Boyle et al. 1988)

$\phi(L, z)=\frac{\phi^{\star}}{L^{\star}(z)\left[\left(\frac{L}{L^{\star}(z)}\right)^{\beta_{1}}+\left(\frac{L}{L^{\star}(z)}\right)^{\beta_{2}}\right]}$,

where $\beta_{1}$ and $\beta_{2}$ are the faint- and bright-end of the luminosity function. A few functional forms have been adopted for the redshift evolution of the break luminosity $L^{\star}(z)$. Using a sample of over 6000 QSOs with $0.35<z<2.3$, Boyle et al. (2000) find that the evolution is well fitted by a second-order polynomial of the form

$L^{\star}(z)=L^{\star}(0) 10^{k_{1} z+k_{2} z^{2}}$.

At redshifts $z \sim 2$, the luminosity evolution stops and the comoving number density of QSOs remains constant up to $z \sim 3$ (Boyle et al. 1988). At $z>3$ the QSO number density declines dramatically. A recent study by Fan et al. (2001) on a sample of 39 high-redshift QSOs from the Sloan Digital Sky Survey, suggests that the number density declines as $\mathrm{e}^{-1.15 z}$ in the redshift range $3.6<z<5$.

Boyle et al. (2000) provide the best-fitting parameters of the $B$-band luminosity function for the two cosmologies adopted in this paper. The $B$-band proper emissivity can then be derived through the integral

$$
\begin{aligned}
\epsilon\left(\nu_{B}, z\right) & =(1+z)^{3} \int_{L_{\min }}^{\infty} L \phi(L, z) \mathrm{d} L \\
& =(1+z)^{3} 10^{k_{1} z+k_{2} z^{2}} \epsilon\left(\nu_{B}, 0\right),
\end{aligned}
$$

where the factor $(1+z)^{3}$ is used to transform comoving into proper number densities. For the flat Einstein-De Sitter universe and the $\Lambda$-cosmology, we have derived $\epsilon\left(\nu_{B}, 0\right)=1.2 \times 10^{24}$ and $7.1 \times$ $10^{23} h_{70} \mathrm{erg} \mathrm{s}^{-1} \mathrm{~Hz}^{-1} \mathrm{Mpc}^{-3}$, respectively. $L_{\min }$ scales with $z$ as $L^{\star}$ and is chosen to correspond to $M_{B}^{\max }=-22$ at $z=3$, for both cosmologies $\left(M_{B}^{\max } \approx-18\right.$ at $z=0$; Madau 1992). Since Eq. (9) well describes a flat luminosity evolution for $z \sim 2$, we have adopted the emissivity of Eq. (10) up to $z=3$. For $z>3$ we have adopted the exponential decline of Fan et al. (2001). 
Boyle et al. (2000) derived the $B$-band luminosity function from observations in the QSOs UV restframe, applying the K-correction for the composite QSO spectrum of Cristiani \& Vio (1990). For consistency, we have used the same spectrum to derive the UV emissivity for $\lambda>1050 \AA$. For $\lambda<1050 \AA$ we have used a powerlaw, $\epsilon(\nu) \propto \nu^{-1.8}$, as measured on a sample of radio-quiet QSOs observed with HST (Zheng et al. 1997).

We have also derived the QSO emissivity from the work of La Franca \& Cristiani (1997). They fitted the luminosity function on a sample of 326 objects (the Homogeneous Bright QSO Survey) by using a different luminosity evolution. Results obtained with this emissivity are very similar to those for the emissivity discussed above and are not presented in this paper.

\subsection{Galaxy emissivity}

The galactic emissivity in the ionising UV has been derived following the method outlined by Madau et al. (1998). The comoving UV emissivity at $\lambda \geq 1500 \AA$ (rest frame) can be derived from galaxy surveys as a function of the redshift. Because UV light is mainly produced by short-lived OB stars, it is possible to convert the UV emissivity into a star-formation history of the universe. If we assume that the mean luminosity evolution of the galaxies in the universe can be described with a single galactic spectrum compatible with the derived star-formation history, a stellar population synthesis model can be used to derive the emissivity at any wavelength.

We have used the latest version of the Bruzual \& Charlot (1993) stellar population synthesis models, updated with a new set of stellar evolutionary tracks and spectral libraries (Bruzual \& Charlot 2001; see also Liu et al. 2000). A Salpeter (1955) IMF with $0.1<M_{\star} / M_{\odot}<$ 100 has been adopted. We have chosen the quasi-empirical library of stellar spectra for solar metallicity, derived from observations for $\lambda>1150 \AA$ and from model of stellar atmospheres for $\lambda<1150 \AA$. The conversion factor between UV luminosity and $S F R$ has been derived from the adopted stellar model, in the limit of continuous starformation (Madau et al. 1998; Kennicutt 1998). For radiation at $\lambda=1500 \AA$ we obtain

$$
\frac{L_{\mathrm{UV}}}{S F R}=7.9 \times 10^{27} \frac{\mathrm{erg} \mathrm{s}^{-1} \mathrm{~Hz}^{-1}}{M_{\odot} \mathrm{yr}^{-1}} \text {. }
$$

Conversion factors for other choices of the IMF and stellar libraries among those provided by the Bruzual \& Charlot model typically differ over a range of $\approx 0.3 \mathrm{dex}$. The same span is also observed when comparing conversion factors obtained with different models (Kennicutt 1998).

The star-formation history at $z<2$ has been calibrated on the UV comoving emissivities tabulated by Madau et al. (1998) for a flat $\Omega_{\mathrm{m}}=1$ universe. Emissivities at $\lambda=2800 \AA$ have been derived from the Canada-France Redshift Survey (Lilly et al. 1996) in the range $0.2<z<1$ and from a HDF-north sample of objects with optical photometric redshifts for $1<z<2$ (Connolly et al. 1997). Madau et al. (1996) have derived the emissivity at $\lambda=1500 \AA$ from objects selected in two redshift ranges at $z \sim 3$ and $z \sim 4$, from object selected on the HDF-north with the UV dropout technique. The lower emissivities at $z>2$ suggested that the star formation rate has reached a maximum at $z \sim 1-2$. However, a different picture emerged from the ground-based survey of Steidel et al. (1999). They selected Lyman break galaxies on a larger area than the HDF, refining the colour selection criteria with spectroscopic observations of a few object in the sample. The derived emissivity (at $\lambda=1700 \AA$ ) for $z \sim 3$ is still consistent with the Madau et al. (1996) value, while the emissivity at $z \sim 4$ does not show a steep decline. A value for the emissivity at $\lambda=1700 \AA$ in the redshift bin $2.5<z<3.5$ can be derived from the luminosity function fitted by Poli et al. (2001) on a combined ground-based and HST database. The datapoints are shown in Fig. 2 as a function of redshift. We have corrected the emissivities of Steidel et al. (1999) and Poli et al. (2001) to include all objects with luminosities from 0 to $\infty$. The large errors in the data derived from Steidel et al. (1999) and Poli et al. (2001) reflect the uncertainties in the faint-end slope $\alpha$ of the Schechter (1976) luminosity function. Steidel et al. (1999) derive $\alpha=-1.60 \pm 0.13$ and Poli et al. (2001) $\alpha=-1.37 \pm 0.20$. In the Madau et al. (1998) tabulation, $\alpha=-1.3$ was used.

A smooth star-formation history has been derived from the observed UV emissivities in Fig. 2, by using Eq. (11) and correcting for dust internal extinction according to the Calzetti's (1997) attenuation law. At high redshift, we have adopted the flat star-formation history suggested by the work of Steidel et al. (1999). Synthetic galactic spectra have then been produced with the Bruzual \& Charlot (2001) code. In Fig. 2 we show the evolution of the modelled UV emissivity at $1500 \AA$ (solid line) and at $2800 \AA$ (dotted line), for a colour excess $E(B-V)=0.1$. The model is compatible with both data at $2800 \AA$ and $z<2$ and data at $1500 \AA$ (and $1700 \AA$ ) and $z>2$. For the chosen $E(B-V)$, the model is also consistent with the evolution of the emissivity in the optical-NIR regime for $z<2$, as tabulated by Madau et al. (1998, not shown in Fig. 2).

Unfortunately, it has not been possible to repeat the same procedure to model the emissivity (and starformation history) in $\Lambda$-cosmology. Only Poli et al. (2001) present a luminosity function derived assuming $\Omega_{\mathrm{m}}=$ $0.3, \Omega_{\Lambda}=0.7$. Steidel et al. (1999) give the emissivity for the objects visible in their survey, but they do not provide a luminosity function for an extrapolation to fainter luminosities. Therefore, we have used the model of the emissivity for $\Omega_{\mathrm{m}}=1$ and scaled it with a redshift-dependent correction: for sufficiently small redshift bin, it can be shown that the ratio of the emissivities in the flat $\Lambda$ - and Einstein-De Sitter cosmologies is $\sqrt{0.7+0.3(1+z)^{3}} /(1+$ $z)^{1.5}$. The emissivity derived in this way is consistent with the data of Poli et al. (2001). Because of Eq. (11), the same ratios applies to the star-formation histories. 


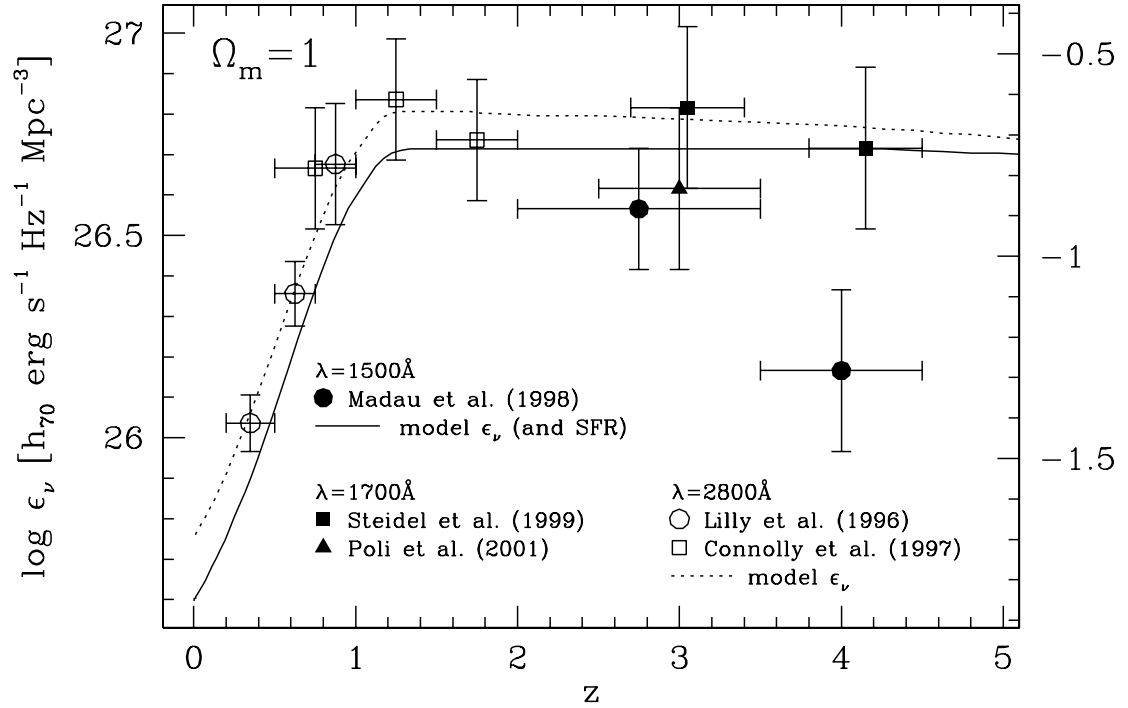

Fig. 2. Comoving UV emissivity in a flat $\Omega_{\mathrm{m}}=1$ universe, from literature (dots) and from the spectral synthesis model described in Sect. 2.3 (lines). The model emissivity has been corrected for internal dust extinction, using the Calzetti's (1997) attenuation law with $E(B-V)=0.1$. Because of Eq. (11) and of the redshift-independent dust correction, emissivity at $\lambda=1500 \AA$ is proportional to the $S F R$ history adopted in the model (scale on right ordinate).
The synthetic spectrum has then been used to derive the emissivity for the ionising UV. Due to the absence of observations, synthetic spectra rely on models of stellar atmospheres for $\lambda \leq 912 \AA$. Charlot \& Longhetti (2001) compared stellar spectra from different models and concluded that discrepancies on the ionising flux are not higher than 0.1 dex. The uncertainty on our modelled emissivity also depends on the uncertainties in the determination of the $S F R$. To quantify the uncertainties in the adopted model, we have computed the effect on the emissivity of the variation of the basic ingredients of the Bruzual \& Charlot (2001) model (IMF, metallicity, stellar libraries). We have used the same description for the UV emissivity at $1500 \AA$ as in the main model (solid line in Fig. 2) and converted it into a star-formation history by using a conversion factor appropriate for the selected IMF and stellar spectra. The synthetic spectra obtained in this way typically differ by less than 0.2 dex at the ionisation limit and 0.3 dex at $600 \AA$.

Spectra at $\lambda \leq 912 \AA$ also need to be corrected for the internal absorption by the galaxy interstellar medium. We describe this correction with the parameter $f_{\text {esc }}$, i.e. the fraction of Lyman-continuum photons that can escape into the IGM without being absorbed by the interstellar medium, either gas or dust. A wide range of values can be found in the literature for $f_{\text {esc }}$, derived both from models of radiative transfer and observations of $\mathrm{H}$ I recombination lines $\left(5 \%<f_{\text {esc }}<60 \%\right.$; for a review, see Barkana \& Loeb 2001). UV observations of local starbursts suggest $f_{\text {esc }} \approx 5 \%$ (Leitherer et al. 1995; Hurwitz et al. 1997; Heckman et al. 2001). Steidel et al. (2001) analysed a composite spectrum of 29 Lyman-break galaxies at $z \sim 3.4$. They derived a ratio between the flux densities at $1500 \AA$ and $900 \AA f_{1500} / f_{900}=4.6 \pm 1$, after correcting for the differential absorption due to the intervening IGM. The $f_{1500} / f_{900}$ ratio for the unattenuated synthetic spectrum that we have used is very similar, $f_{1500} / f_{900} \approx 5.3$. If we assume that $40 \%$ of the radiation at $1500 \AA$ is absorbed by dust (as obtained from the Calzetti's attenuation law with $E(B-V)=0.1)$, the observed $f_{1500} / f_{900}$ ratio is equivalent to $f_{\mathrm{esc}} \approx 0.4$ (if the internal absorption in the Lyman continuum do not change significantly with $\lambda$ ). Because of the increase of the disk density with the redshift, $f_{\text {esc }}$ is expected to decrease with $z$; it is also found to depend heavily on the details of the distribution of the sources and the gas, i.e. whether the stars and/or gas are clumped or not (Wood \& Loeb 2000). In this work, we will use a wavelength and redshift independent $f_{\text {esc }}$, by which we multiply the synthetic spectrum at $\lambda \leq 912 \AA$. We will show results for $f_{\text {esc }}=0.05$ and 0.40 , to cover the range of values suggested by local and $z \sim 3.4$ observations, and for an intermediate value, $f_{\text {esc }}=0.1$.

Finally, the galactic emissivity has been converted from comoving to proper, multiplying by $(1+z)^{3}$. The total emissivity in Eq. (1) is the sum of the QSOs and the galaxy contribution.

\section{The ionising background at $912 \AA$}

In Fig. 3 we show the modelled UV background, $J(\nu, z)$, at the Lyman limit as a function of redshift (solid lines) for the flat universe with $\Omega_{\mathrm{m}}=1$. The total background is shown as the sum of the QSO contribution (the same in each model; dotted line) and the galaxy contribution (scaled with $f_{\text {esc }}$; dashed lines). As predicted by other authors, for large values of $f_{\text {esc }}$ the ionising background produced by galaxies dominates over the flux from QSOs (Giallongo et al. 1997; Devriendt et al. 1998; Shull et al. 1999).

At high redshift, the value of the UV background is constrained by the analysis of the proximity effect, i.e. the decrease in the number of intervening absorption lines that is observed in a QSO spectrum when approaching the QSO's redshift (Bajtlik et al. 1988). Using high resolution spectra, Giallongo et al. (1996) derived $J(912 \AA)=5.0_{-1}^{+2.5} \times 10^{-22} \mathrm{erg} \mathrm{cm}^{-2} \mathrm{~s}^{-1} \mathrm{~Hz}^{-1} \mathrm{sr}^{-1}$ for $1.7<z<4.1$ (see also Giallongo et al. 1999). Larger values are obtained by Cooke et al. (1997), 


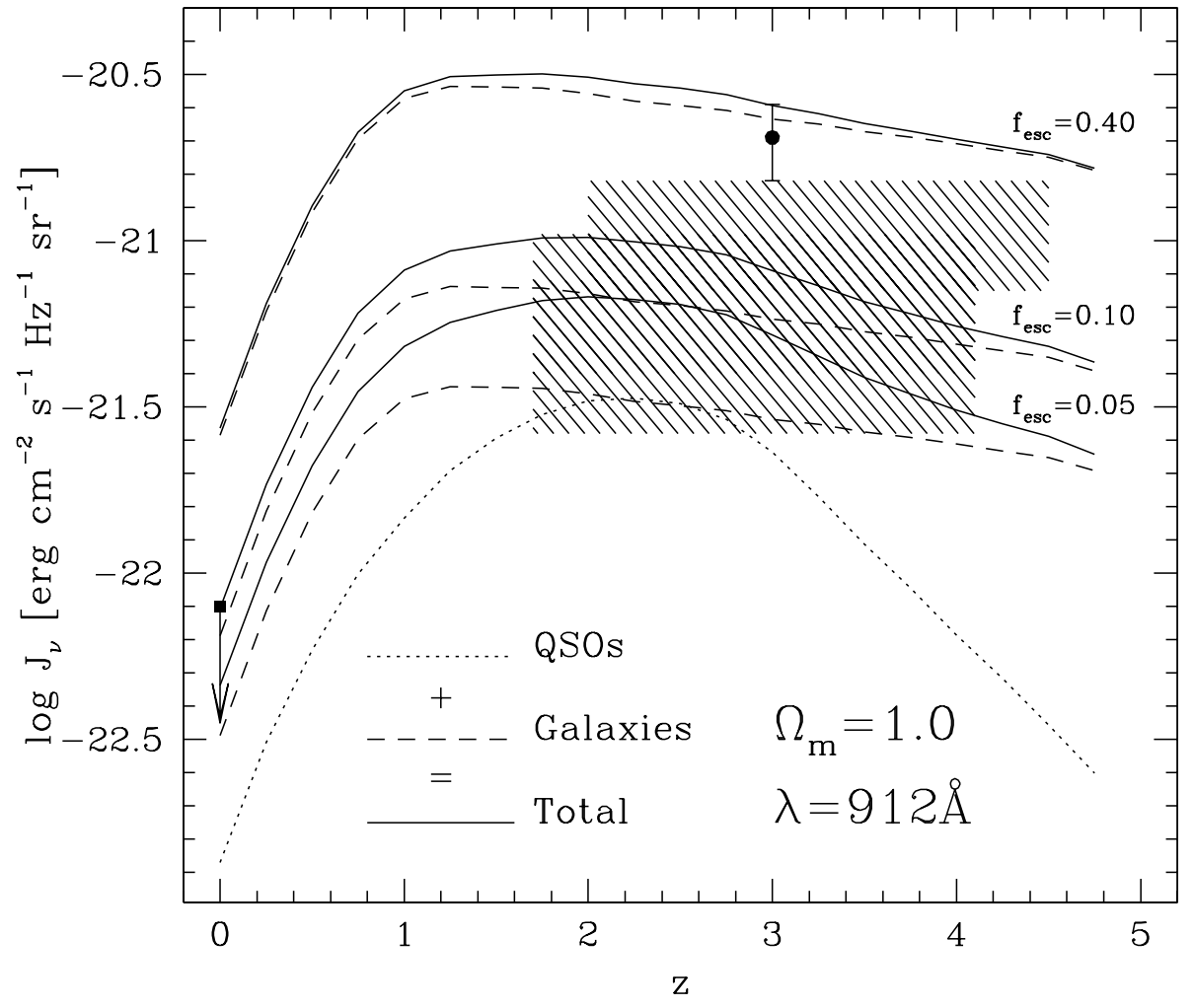

Fig. 3. UV background at $\lambda=912 \AA$ for the models with $f_{\text {esc }}=0.05,0.1$ and 0.4 (solid lines), in a flat $\Omega_{\mathrm{m}}=1$ universe. Also shown are the separate contribution of the QSOs (dotted line) and of the galaxies (dashed lines, each corresponding to a value of $f_{\text {esc }}$ ). The shaded area refer to the Lyman limit UV background estimated from the proximity effect (Giallongo et al. 1996; Cooke et al. 1997; Scott et al. 2000). The arrow shows an upper limit for the local ionising background (Vogel et al. 1995). The datapoint at $z=3$ is derived from a composite spectrum of Lyman-break galaxies (Steidel et al. 2001). The models and the datapoint of Steidel et al. (2001) have been multiplied by a $z$ dependent factor, to take into account the cloud emission (see text).
$J(912 \AA)=1.0_{-0.3}^{+0.5} \times 10^{-21} \mathrm{erg} \mathrm{cm}^{-2} \mathrm{~s}^{-1} \mathrm{~Hz}^{-1} \mathrm{sr}^{-1}$ for $2.0<z<4.5$. A recent re-analysis of moderate resolution spectra by Scott et al. (2000) has lead to $J(912 \AA)=$ $7.0_{-4.4}^{+3.4} \times 10^{-22} \mathrm{erg} \mathrm{cm}^{-2} \mathrm{~s}^{-1} \mathrm{~Hz}^{-1} \mathrm{sr}^{-1}$ for the same redshift range. We show these measurements in Fig. 3 with a shaded area: the spread of the measurements obtained with different methods and data gives an idea of the uncertainties associated with the study of the proximity effect.

Measurements of the ionising background at low redshift are not less uncertain. Kulkarni \& Fall (1993) reported a first tentative detection of the proximity effect in a sample of 13 QSOs at $z<1$ observed with HST. They obtained $J(912 \AA)=6_{-4}^{+30} \times$ $10^{-24} \mathrm{erg} \mathrm{cm}^{-2} \mathrm{~s}^{-1} \mathrm{~Hz}^{-1} \mathrm{sr}^{-1}$, the large uncertainties due to the small number of available absorbers. Vogel et al. (1995) derived a $2 \sigma$ upper limit $J(912 \AA)<8.0 \times$ $10^{-23} \mathrm{erg} \mathrm{cm}^{-2} \mathrm{~s}^{-1} \mathrm{~Hz}^{-1} \mathrm{sr}^{-1}$, by studying $\mathrm{H} \alpha$ emission in a high latitude Galactic cloud. This upper limit is shown in Fig. 3.

We must remember here that our model does not take into account the Lyman-continuum emission from recombination in Ly $\alpha$ clouds. Haardt \& Madau (1996) have shown that radiative recombination provides an important contribution to the ionising background. Using only QSOs as source of ionising radiation, they obtain $J(912 \AA)=5 \times 10^{-22} \mathrm{erg} \mathrm{cm}^{-2} \mathrm{~s}^{-1} \mathrm{~Hz}^{-1} \mathrm{sr}^{-1}$ at $z=2.5$, well within the shaded area in Fig. 3. Similar results are obtained by Fardal et al. (1998). Although the cloud contribution to the background depends on the adopted emissivity and intergalactic absorption, we have obtained a rough estimate of its importance by using UV background spectra kindly provided by F. Haardt. At $z=3$, the models of Haardt \& Madau (1996) are a factor 1.7 higher than for the case of a purely absorbing medium; at $z=0$, the factor reduces to 1.3. A simple linear interpolation between these two points reproduces the actual data for $0<z<5$ within 5\%. The models shown in Fig. 3 are multiplied for this $z$-dependent factor.

Several models can produce a value of $J(912 \AA)$ compatible with one of the measurements from the proximity effect at $z \sim 3$ shown in Fig. 3, from a a simple QSO-dominated background to models with $f_{\text {esc }} \sim 0.2$. However, a more stringent condition, $f_{\text {esc }} \lesssim 0.10$, is required not to exceed the local upper limit of Vogel et al. (1995). Steidel et al. (2001) used their composite spectrum of Lyman-break galaxies and the UV emissivities of Steidel et al. (1999) to derive the ionising flux at $z=3$. They obtained $J(912 \AA)=1.2 \pm 0.3 \times$ $10^{-21} \mathrm{erg} \mathrm{cm}^{-2} \mathrm{~s}^{-1} \mathrm{~Hz}^{-1} \mathrm{sr}^{-1}$, a value consistent with our model for $f_{\text {esc }}=0.4$. If Lyman-break galaxies with a spectrum similar to that observed by Steidel et al. (2001) dominate the UV background, they will produce an ionising flux higher than the local and high-redshift estimates.

Similar results are obtained in the $\Omega_{\mathrm{m}}=0.3, \Omega_{\Lambda}=0.7$ universe (not shown). Because of Eq. (2) and of the factor we have used in Sect. 2.3 to derive the emissivity for the $\Lambda$-cosmology, the contribution of galaxies to the emissivity is exactly the same in both the universe models adopted here. The QSOs contribution, instead, depends on an independent fit of the luminosity function (Sect. 2.2). In the new cosmology, the UV background produced by QSOs is slightly larger than the value for the 


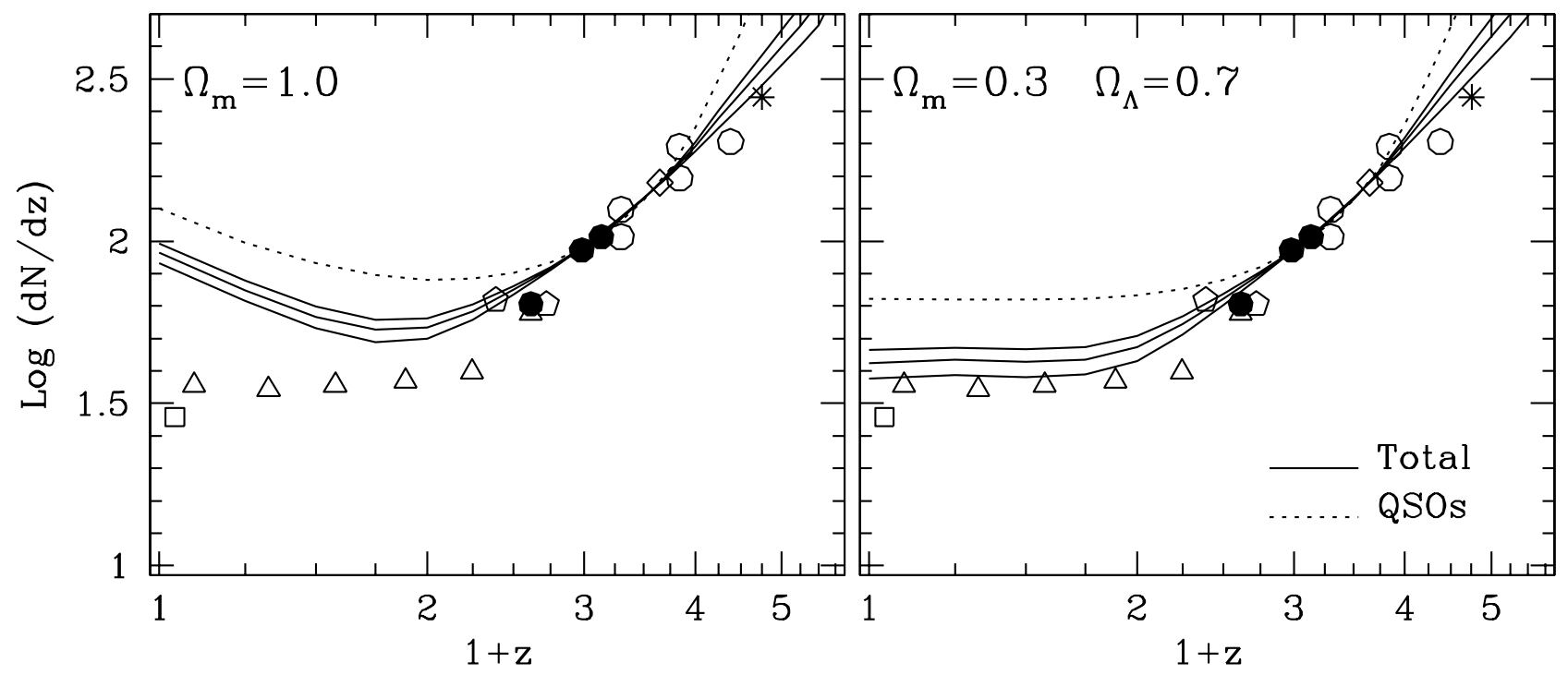

Fig. 4. Number density evolution of the Ly $\alpha$ forest with $N_{\mathrm{H}}=10^{13.64-16} \mathrm{~cm}^{-2}$, for the two cosmologies adopted in this paper. Dotted lines refer to the evolution compatible with an ionising UV background due only to QSOs. Solid lines show the evolution when both QSOs and galaxies contribute to the background, for the models with $f_{\text {esc }}=0.05$ (upper line), 0.1 and 0.4 (lower line). Data points come from several observations in the literature for the column density range $N_{\mathrm{H}}=10^{13.64-16} \mathrm{~cm}^{-2}$, as given by Kim et al. (2001). The modelled evolution has been normalized to the observed evolution in the redshift range $2<z<3$.

Einstein-De Sitter model, by about $30 \%$ at the peak of the QSOs contribution. ${ }^{1}$

\section{The evolution of the Lyman forest}

Further constraints can be obtained by studying the effect of the evolution of the ionising background on the evolution of absorbers. Davé et al. (1999) have studied the evolution of the low-redshift Ly $\alpha$ forest in a hydrodynamic cosmological simulation, adopting an UV ionising background with the same redshift evolution as that of Haardt \& Madau (1996). They found a sharp transition at $z=1.7$ for the number density evolution, $\mathrm{d} N / \mathrm{d} z$. The change in evolution is primarily due to the drop in the UV ionising background, resulting from the decline in the QSO population. The formation of structure by gravitational growth plays only a minor role in the evolution. In the absence of structure evolution, it is possible to derive an analytical approximation for the evolution of $\mathrm{d} N / \mathrm{d} z$ with $J_{\nu}$ and the Hubble expansion. For clouds in photoionisation equilibrium with the background, it is easy to show that the evolution of lines above a given threshold in column density can be written as (Davé et al. 1999)

$$
\left(\frac{\mathrm{d} N}{\mathrm{~d} z}\right)_{N_{\mathrm{H}_{\mathrm{I}}>N_{\mathrm{H} \mathrm{I}}}} \propto \frac{1}{H(z)}\left[\frac{(1+z)^{5}}{\Gamma_{\mathrm{H}_{\mathrm{I}}}(z)}\right]^{\beta-1},
$$

${ }^{1}$ As pointed out by the referee, the background is, in principle, independent on the adopted cosmology. The slight difference between the two QSO backgrounds arises because of the cosmology-dependent correction for incompleteness in the luminosity function. where $H(z)$ is the Hubble parameter and $\beta$ the coefficient of the power-law distribution of clouds with column density (Eq. (7)). $\Gamma_{\mathrm{H}_{\mathrm{I}}}(z)$ is the photo-ionisation rate

$$
\Gamma_{\mathrm{HI}}(z)=\int_{\nu_{0}}^{\infty} \frac{4 \pi J(\nu, z)}{h \nu} \sigma_{\mathrm{HI}}(\nu) \mathrm{d} \nu
$$

with $\nu_{0}$ the frequency of the Lyman limit and $\sigma_{\mathrm{HI}}(\nu)=$ $\sigma_{\mathrm{HI}}\left(\nu_{0} / \nu\right)^{3}$ the $\mathrm{H}$ I photo-ionisation cross-section.

In Fig. 4 we show $\mathrm{d} N / \mathrm{d} z$ for Lyman forest clouds in the column density range $N_{\mathrm{HI}}=10^{13.64-16} \mathrm{~cm}^{-2}$. Data points come from several sources in the literature and from new high resolution VLT/UVES spectra of three QSO (Kim et al. 2001). For each of our models, we have computed $\Gamma_{\mathrm{HI}}(z)$ and we have derived the evolution of $\mathrm{d} N / \mathrm{d} z$ according to Eq. (12), for the two cosmologies adopted in this paper. The evolution has been normalized to the observed values for $2<z<3$. In this redshift range, the UV background is nearly flat for any of the models and it is easy to show, from Eq. (12), that $\mathrm{d} N / \mathrm{d} z \propto(1+z)^{5 \beta-6.5}$, independently of the cosmology. By fitting the observed data, Kim et al. (2001) have derived $\mathrm{d} N / \mathrm{d} z \propto(1+z)^{2.19}$ for $z>1.5$. A value $\beta \approx 1.7$ can well reproduce the evolution derived from the observations. This is consistent with fits of the density distribution, that give $\beta=1.68 \pm 0.15$ over $N_{\mathrm{H}}=10^{14-16} \mathrm{~cm}^{-2}$ at $z \sim 1$ (Kim et al. 2001). On the other hand, weaker lines $\left(N_{\mathrm{H} \text { I }} \lesssim 10^{14} \mathrm{~cm}^{-2}\right)$ are known to have a flatter distribution in column density $(\beta \sim 1.4-1.5$; Giallongo et al. 1996; Kim et al. 2001). This will produce a slower redshift evolution, as observed in this column density range for $z>1.5(\gamma \sim 1$; Kim et al. 2001). We remind here that in Sect. 2.1 we have used $\beta=1.46$, that provides a good description of the density distribution over a much larger column density range. 
The analysis of Kim et al. (2001) shows that the change in evolution occurs at $z \approx 1$, rather than at $z \approx 1.7$, as previously suggested (Weymann et al. 1998). In Fig. 4 the break at $z \approx 1$ can be reproduced if the contribution of galaxies to the background is dominant. This is because of the rapid decrease of the star-formation rate (and of $\left.\Gamma_{\mathrm{HI}}(z)\right)$ below this redshift (Fig. 2). The photo-ionisation rate of a pure QSOs background, instead, peaks at $z \sim 2.5$ and has a slower evolution with $z$. It is interesting to note that the modelled evolution is closer to the observed data for the $\Lambda$-cosmology. For the Einstein-De Sitter universe, $\mathrm{d} N / \mathrm{d} z$ grows for $z<1$, which is not observed. However, the discrepancy may be mitigated when the effect of the formation of structures on $\mathrm{d} N / \mathrm{d} z$ is taken into account (Davé et al. 1999).

The modelled $\mathrm{d} N / \mathrm{d} z$ do not depend on our approximation of a purely absorbing intergalactic medium, since the contribution of cloud emission to $\Gamma_{\mathrm{HI}}(z)$ is nearly constant with redshift (see Fig. 6 in Haardt \& Madau 1996).

\section{Conclusions}

In this work we have derived the H I-ionising background, resulting from the integrated contribution of QSOs and galaxies, taking into account the opacity of the intervening IGM. We have modelled the IGM with pure-absorbing clouds, with a distribution in column density of neutral hydrogen, $N_{\mathrm{H} \text { I }}$, and redshift, $z$, derived from recent observations of the Ly $\alpha$ forest (Kim et al. 2001) and from Lyman Limit systems. The QSOs emissivity has been derived from the recent fits of Boyle et al. (2000), while we have used the stellar population synthesis model of Bruzual \& Charlot (2001) and a star-formation history from UV observations for the galaxy emissivity. Due to the present uncertainties in models and observations, we have used three values for the fraction of ionising photons that can escape a galaxy interstellar medium, $f_{\mathrm{esc}}=0.05$, 0.1 and 0.4 , as suggested by local and high- $z$ UV observations of galaxies, respectively.

The contribution of galaxies to the UV background is found to be comparable or larger than that of QSOs. This is consistent with other determinations of the galactic contribution to the background (Giallongo et al. 1997; Devriendt et al. 1998; Shull et al. 1999). Taking into account (in a rough form) the contribution of reemission from the IGM clouds (Haardt \& Madau 1996; Fardal et al. 1998 ), we find that all models with $0<f_{\text {esc }} \lesssim 0.10$ can provide an ionising background within the limits measured from observations of the proximity effect at $z \sim 3$ and not exceeding the upper limit for the local background at $z=0$.

The analysis of Kim et al. (2001) shows that the break in the evolution of the Ly $\alpha$ forest, $\mathrm{d} N / \mathrm{d} z$, occurs at $z \sim 1$. We used the evolution of the ionising background in our model to derive $\mathrm{d} N / \mathrm{d} z$, assuming that the formation of structure plays only a minor role (Davé et al. 1999). The rapid decrease of star-formation for $z \lesssim 1$ can easily explain the observed break, while a QSO-only background would produce a break at $z \sim 2$ (Davé et al. 1999). For the ionising background to have an evolution similar to that of a galaxy-only model, high values of $f_{\text {esc }}$ are needed. This result apparently pushes the galaxy contribution in an opposite direction with respect to the estimates of the absolute value of the ionising background. The flat evolution of $\mathrm{d} N / \mathrm{d} z$ at $z<1$ is much better reproduced adopting a $\Lambda$-cosmology $\left(\Omega_{\mathrm{m}}=0.3, \Omega_{\Lambda}=0.7\right)$ rather than a $\Omega_{\mathrm{m}}=1$ Einstein-De Sitter universe. However, such a result needs to be confirmed after properly taking the formation of structure into account.

A significant contribution from galaxies to the ionising metagalactic flux would correspond to a softening of its spectrum with respect to a purely QSO-dominated background. This effect would be particularly important at very high and very low redshift. Observations of the evolution of the Si IV to C IV ratio with redshift (Savaglio et al. 1997; Songaila 1998) seem to confirm the progressive softening of the UV background at $z>3$.

To summarise our result, a galaxy-dominated background with $f_{\text {esc }} \lesssim 0.1$ is consistent with the estimates of $J(912 \AA)$. In the hypothesis that the formation of structures plays a negligible effect, $f_{\text {esc }} \gtrsim 0.05-0.1$ is needed in order to explain the observed $\mathrm{d} N / \mathrm{d} z$ of Ly $\alpha$ absorbers. The rapidly improving knowledge derivable from numerical simulations, the determination of the cosmological evolution of the Lyman forest, the proximity effect estimates of the ionising background, and the evolution of the intensity ratios of metal absorption lines will put soon constraints on the relative galaxy/QSO contribution to the UV background and, together with direct measurements of the Lyman continuum emission from galaxies, will make it possible to address the issue of the evolution of the escaping fraction of photons from galaxies as a function of $z$.

Acknowledgements. We are greatly indebted to F. Haardt (the referee) and P. Madau for providing us with results from their cosmological radiative transfer code prior to publication and for enlightening comments; and to S. Charlot for suggestions and help with the use of the latest version of the GISSEL code. We also acknowledge stimulating discussion with S. D'Odorico, B. Ciardi, A. Ferrara and A. Grazian. This work was partially supported by the Research and Training Network "The Physics of the Intergalactic Medium" set up by the European Community under the contract HPRN-CT200000126 RG29185.

\section{References}

Bajtlik, S., Duncan, R. C., \& Ostriker, J. P. 1988, ApJ, 327, 570

Barkana, R., \& Loeb, A. 2001, Physics Reports, in press

Boyle, B. J., Shanks, T., Croom, S. M., et al. 2000, MNRAS, 317,1014

Boyle, B. J., Shanks, T., \& Peterson, B. A. 1988, MNRAS, 235, 935

Bruzual, A. G., \& Charlot, S. 1993, ApJ, 405, 538

Bruzual, A. G., \& Charlot, S. 2001, in preparation 
Calzetti, D. 1997, in The Ultraviolet Universe at Low and High Redshift, ed. W. H. E. A. Waller (New York: American Institute of Physics), AIP Conf. Proc., 408, 403

Charlot, S., \& Longhetti, M. 2001, MNRAS, in press

Connolly, A. J., Szalay, A. S., Dickinson, M., Subbarao, M. U., \& Brunner, R. J. 1997, ApJ, 486, L11

Cooke, A. J., Espey, B., \& Carswell, R. F. 1997, MNRAS, 284, 552

Cristiani, S., \& Vio, R. 1990, A\&A, 227, 385

Davé, R., Hernquist, L., Katz, N., \& Weinberg, D. H. 1999, ApJ, 511, 521

Devriendt, J. E. G., Sethi, S. K., Guiderdoni, B., \& Nath, B. B. 1998, MNRAS, 298, 708

Fan, X., Strauss, M., Schneider, et al. 2001, AJ, 121, 31

Fardal, M. A., Giroux, M. L., \& Shull, J. M. 1998, AJ, 115, 2206

Freedman, W. L., Madore, B. F., Gibson, B. K., et al. 2001, ApJ, 553, 47

Giallongo, E., Cristiani, S., D’Odorico, S., Fontana, A., \& Savaglio, S. 1996, ApJ, 466, 46

Giallongo, E., Fontana, A., Cristiani, S., \& D’Odorico, S. 1999, ApJ, 510, 605

Giallongo, E., Fontana, A., \& Madau, P. 1997, MNRAS, 289, 629

Haardt, F., \& Madau, P. 1996, ApJ, 461, 20

Heckman, T., Sembach, K. R., Meurer, G., et al. 2001, ApJ, in press

Hurwitz, M., Jelinsky, P., \& Dixon, W. V. D. 1997, ApJ, 481, L31

Kennicutt, R. C. 1998, ARA\&A, 36, 189

Kim, T. S., Cristiani, S., \& D'Odorico, S. 2001, A\&A, in press

Kulkarni, V. P., \& Fall, S. M. 1993, ApJ, 413, L63

La Franca, F., \& Cristiani, S. 1997, AJ, 113, 1517

Leitherer, C., Ferguson, H. C., Heckman, T. M., \& Lowenthal, J. D. 1995, ApJ, 454, L19

Lilly, S. J., Le Fevre, O., Hammer, F., \& Crampton, D. 1996, ApJ, 460, L1
Liu, M. C., Charlot, S., \& Graham, J. R. 2000, ApJ, 543, 644 Machacek, M. E., Bryan, G. L., Meiksin, A., et al. 2000, ApJ, 532,118

Madau, P. 1991, ApJ, 376, L33

Madau, P. 1992, ApJ, 389, L1

Madau, P., Ferguson, H. C., Dickinson, M. E., et al. 1996, MNRAS, 283, 1388

Madau, P., Pozzetti, L., \& Dickinson, M. 1998, ApJ, 498, 106 Moller, P., \& Jakobsen, P. 1990, A\&A, 228, 299

Osterbrock, D. E. 1989, Astrophysics of Gaseous Nebulae and Active Galactic Nuclei (Mill Valley: University Science Book)

Paresce, F., McKee, C. F., \& Bowyer, S. 1980, ApJ, 240, 387

Petitjean, P., Webb, J. K., Rauch, M., Carswell, R. F., \& Lanzetta, K. 1993, MNRAS, 262, 499

Poli, F., Menci, N., Giallongo, E., et al. 2001, ApJL, in press

Salpeter, E. E. 1955, ApJ, 121, 161

Savaglio, S., Cristiani, S., D’Odorico, S., et al. 1997, A\&A, 318, 347

Schechter, P. 1976, ApJ, 203, 297

Scott, J., Bechtold, J., Dobrzycki, A., \& Kulkarni, V. P. 2000, ApJS, 130, 67

Shull, J. M., Roberts, D., Giroux, M. L., Penton, S. V., \& Fardal, M. A. 1999, AJ, 118, 1450

Songaila, A. 1998, AJ, 115, 2184

Steidel, C. C., Adelberger, K. L., Giavalisco, M., Dickinson, M., \& Pettini, M. 1999, ApJ, 519, 1

Steidel, C. C., Pettini, M., \& Adelberger, K. L. 2001, ApJ, 546, 665

Storrie-Lombardi, L. J., McMahon, R. G., Irwin, M. J., \& Hazard, C. 1994, ApJ, 427, L13

Vogel, S. N., Weymann, R., Rauch, M., \& Hamilton, T. 1995, ApJ, 441, 162

Weymann, R. J., Jannuzi, B. T., Lu, L., et al. 1998, ApJ, 506, 1

Wood, K., \& Loeb, A. 2000, ApJ, 545, 86

Zheng, W., Kriss, G. A., Telfer, R. C., Grimes, J. P., \& Davidsen, A. F. 1997, ApJ, 475, 469 